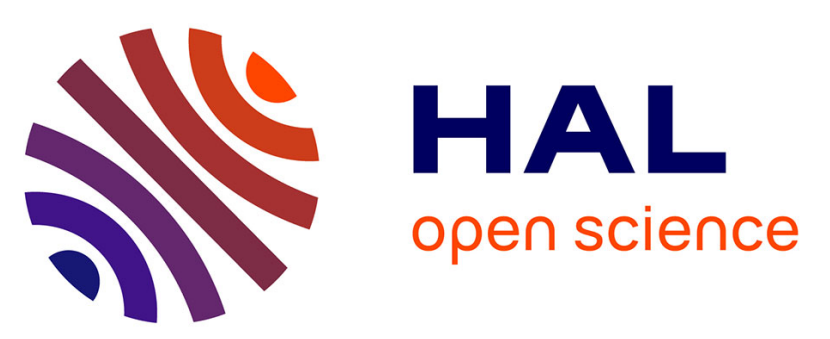

\title{
Relaxing the conditions for parameter estimation-based observers of nonlinear systems via signal injection
}

Bowen Yi, Roméo Ortega, Weidong Zhang

\section{To cite this version:}

Bowen Yi, Roméo Ortega, Weidong Zhang. Relaxing the conditions for parameter estimation-based observers of nonlinear systems via signal injection. Systems and Control Letters, 2018, 111, pp.18-26. 10.1016/j.sysconle.2017.10.011 . hal-01816429

\section{HAL Id: hal-01816429}

https://hal-centralesupelec.archives-ouvertes.fr/hal-01816429

Submitted on 17 Jun 2020

HAL is a multi-disciplinary open access archive for the deposit and dissemination of scientific research documents, whether they are published or not. The documents may come from teaching and research institutions in France or abroad, or from public or private research centers.
L'archive ouverte pluridisciplinaire HAL, est destinée au dépôt et à la diffusion de documents scientifiques de niveau recherche, publiés ou non, émanant des établissements d'enseignement et de recherche français ou étrangers, des laboratoires publics ou privés. 


\title{
Relaxing the Conditions for Parameter Estimation-Based Observers of Nonlinear Systems via Signal Injection
}

\author{
Bowen $\mathrm{Yi}^{\mathrm{a}}$, Romeo Ortega ${ }^{\mathrm{b}}$, Weidong Zhang,c \\ ${ }^{a}$ Department of Automation, Shanghai Jiao Tong University, Shanghai 200240, China \\ ${ }^{b}$ Laboratoire des Signaux et Systèmes, CNRS-CentraleSupélec, Plateau du Moulon, Gif-sur-Yvette 91192, France \\ ${ }^{c}$ School of Mechatronic Engineering and Automation, Shanghai University, Shanghai 200444, China
}

\begin{abstract}
Parameter estimation-based observers are a new kind of state reconstruction methods where the state observation task is translated into an on-line parameter estimation problem. A key step for its application is the transformation of the system dynamics into a particular cascade form, which involves the solution of a partial differential equation that, moreover, should satisfy some injective requirement. In this note we use a recently proposed technique of signal injection to generate new outputs and simplify these tasks. In this way, we make this observer applicable to a wider class of nonlinear systems - even with indistinguishable states. The application of the proposed approach is illustrated with the design of a novel sensorless controller for magnetic levitation systems.
\end{abstract}

Keywords: Signal Injection; Nonlinear Systems; Observer; Parameter Estimation.

\section{Introduction}

In the last three decades we have witnessed rapid progress of nonlinear observer theory in the control literature. Recent reviews may be found in [4, 5, 10. The first systematic observer theory for nonlinear systems was developed in [18]. In this seminal work, a nonlinear state transformation to obtain a linear system up to an output injection was proposed. The approach has some extremely restrictive conditions, stymying its applicability for most physical systems. Since then various approaches to design observers for nonlinear systems have been pursued, e.g., based on nonlinearity domination via high-gain [11, 8], on the generation of invariant manifolds [4, 14], on passivity and dissipativity theory [3] and on optimization theory [1]. Particular attention has been given to the Kazantzis-KravarisLuenberger (KKL) observer [15], which is the extension of Luenberger's cornerstone work [19] for linear time-invariant (LTI) systems to the nonlinear case. For autonomous systems, KKL observers have been systematically studied from both theoretical and numerical aspects [17, 2]. However, the existence of KKL observer and its design procedure for nonlinear systems with inputs are, to the best of our knowledge, still open problems.

In 22] a novel class of observers for nonlinear control systems, called parameter estimation-based observers (PEBOs), was proposed. The distinguishing feature of PEBOs is that it formulates the observer design problem as a parameter estimation problem. A key step for the application of PEBO is the transformation of the system dynamics into a particular cascaded form, a task that requires the solution of a partial differential equation (PDE). Moreover, this solution should define an injective map and an associated (consistent) parameter identification task has to be solved. In this note we propose to use the signal injection technique introduced in [6] to generate new "virtual outputs" that, included in the PEBO design, provide additional degrees of freedom to satisfy the three aforementioned

Email addresses: yibowen@ymail.com (Bowen Yi), ortega@lss.supelec.fr (Romeo Ortega), Corresponding author: wdzhang@sjtu.edu.cn (Weidong Zhang) 
requirements. The inclusion of probing signals for observer and controller design is standard practice in many practical applications like sensorless control of electrical machines [13], vibration control [20] and active islanding detection schemes [12]. It is also routinely used in parameter identification and adaptive control to achieve the persistency of excitation condition required for parameter convergence and control robustification [25].

To apply the signal injection technique in the PEBO scenario it is necessary to extend the results of [6] in three directions.

(i) Extend the theory for scalar systems to the case multi-input-multi-output (MIMO) systems.

(ii) Refine the averaging analysis considering the use in the control of the estimated virtual output instead of the virtual output itself.

(iii) Redesign the estimator of an alternative virtual output instead of the one obtained for the nominal system.

Another contribution of the paper is to propose a technique to solve the PDE using mappings pseudo inverses and Poincare's Lemma. This new procedure, which translates the problem of solution of the PDE into the solvability of a set of (nonlinear) algebraic equations and an, easily verifiable, integrability condition-both steps some free mappings - is more systematic and provides additional degrees of freedom to solve the problem.

The remaining of paper is organized as follows. In Section 2, a brief review of PEBO is given. The main idea and a motivating example are presented in Section 3 . Sections 4 and 5 describe the use of signal injection in PEBO, while in Section 6 we discuss the new technique to solve the PDE. Examples that illustrate the main results of the paper as given in Section 7. Section 8 contains the conclusion and future work. To enhance readability the technically involved proofs are given in appendices.

Notations. $\mathbb{O}=\operatorname{col}(0, \ldots, 0) . \mathbb{1}=\operatorname{col}(1, \ldots, 1) . A^{\dagger}$ denotes the generalized inverse of the matrix $A$. All the functions are supposed smooth enough. Given a function $f: \mathbb{R}^{n} \rightarrow \mathbb{R}$ we define $\partial_{i} f:=\frac{\partial f}{\partial x_{i}}$ and the differential operator $\nabla f:=\left(\frac{\partial f}{\partial x}\right)^{\top}$. For a mapping $F: \mathbb{R}^{n} \rightarrow \mathbb{R}^{m}$ we define the $i j$-th element of its $n \times m$ (transposed) Jacobian matrix as $(\nabla F)_{i j}:=\frac{\partial F_{j}}{\partial x_{i}}$. $\mathcal{O}$ is the "uniform big O" symbol, that is, $f(z, \varepsilon)=\mathcal{O}(\varepsilon)$ if and only if $|f(z, \varepsilon)| \leq C \varepsilon$ for a constant $C$ independent of $z$ and $\varepsilon$.

\section{Parameter Estimation-based Observers}

To apply the signal injection technique it is necessary to develop a slight variation of the main result of [22], where the full state - instead of part of it as done in [22] —is observed. For the sake of completeness we give also the proof of this result.

Proposition 1. Consider the nonlinear system

$$
\begin{aligned}
& \dot{x}=f(x)+g(x) u \\
& y=h(x)
\end{aligned}
$$

where $x \in \mathbb{R}^{n}, y \in \mathbb{R}^{p}, u \in \mathbb{R}^{m}$ and $g(x)$ is full rank, together with the following assumptions.

(A1) (PDE solvability) There exist mappings

$$
\phi: \mathbb{R}^{n} \rightarrow \mathbb{R}^{n_{z}}, \beta: \mathbb{R}^{p} \times \mathbb{R}^{m} \rightarrow \mathbb{R}^{n_{z}},
$$

with $n_{z} \geq n-p$ satisfying the PDE

$$
\nabla^{\top} \phi(x)[f(x)+g(x) u]=\beta(h(x), u)
$$


(A2) (Left invertibility) There exists a mapping $\phi^{L}: \mathbb{R}^{n_{z}} \times \mathbb{R}^{p} \rightarrow \mathbb{R}^{n}$ such that

$$
\phi^{L}(\phi(x), h(x))=x .
$$

(A3) (Consistent identification) There exists mappings

$$
M: \mathbb{R}^{n_{\zeta}} \times \mathbb{R}^{n_{z}} \times \mathbb{R}^{p} \times \mathbb{R}^{m} \rightarrow \mathbb{R}^{n_{\zeta}}, N: \mathbb{R}^{n_{\zeta}} \times \mathbb{R}^{n_{z}} \times \mathbb{R}^{p} \times \mathbb{R}^{m} \rightarrow \mathbb{R}^{n_{z}},
$$

with $n_{\zeta} \in \mathbb{N}_{+}$such that the parameter estimator

$$
\begin{aligned}
\dot{\zeta} & =M(\zeta, \mathfrak{z}, y, u) \\
\hat{\vartheta} & =N(\zeta, \mathfrak{z}, y, u)
\end{aligned}
$$

generates a consistent estimate for the nonlinear regression model

$$
\begin{aligned}
\dot{\mathfrak{z}} & =\beta(y, u) \\
y & =h\left(\phi^{L}(\mathfrak{z}+\vartheta, y)\right),
\end{aligned}
$$

where $\vartheta \in \mathbb{R}^{n_{z}}$ is a vector of constant, unknown parameters. That is, all signals are bounded and

$$
\lim _{t \rightarrow \infty}|\hat{\vartheta}(t)-\vartheta|=0
$$

Under these assumptions, the PEBO

$$
\hat{x}=\phi^{L}(\mathfrak{z}+\hat{\vartheta}, y)
$$

verifies $\lim _{t \rightarrow \infty}|\hat{x}(t)-x(t)|=0$.

Proof 1. Defining the function $z=\phi(x)$, it follows from (2) that

$$
\dot{z}=\beta(h(x), u) .
$$

This, together with (6) and an integration, implies that

$$
z(t)-\mathfrak{z}(t)=\vartheta, \forall t \geq 0,
$$

where $\vartheta:=z(0)-\mathfrak{z}(0)$ is the integration constant. Now, Assumption (A2) ensures that

$$
x=\phi^{L}(z, y)=\phi^{L}(\mathfrak{z}+\vartheta, y),
$$

where we have used (9) to obtain the second identity. Replacing the expression above in the output map yields (7). The proof is completed invoking Assumption (A3) and defining the state estimate $\hat{x}$ by replacing $\vartheta$ in the place of $\vartheta$ in the equation above.

\section{Main Idea and Motivating Example}

Clearly, the design of the PEBO is simplified if additional signals are available for measurement. The main contribution of the paper is to propose a procedure - based on the signal injection technique of [6] - to generate an approximation of the signal $y_{v}=h_{v}(x) \in \mathbb{R}^{p}$, where we defined

$$
h_{v}(x):=\nabla^{\top} h(x) g(x) b
$$

with $b \in \mathbb{R}^{m}$ a free vector utilized in the scaling of the signal injections.

To motivate the addition of this virtual output signal in the context of PEBO we present in this section its application for a benchmark example. Namely, a magnetic levitation system consisting of 


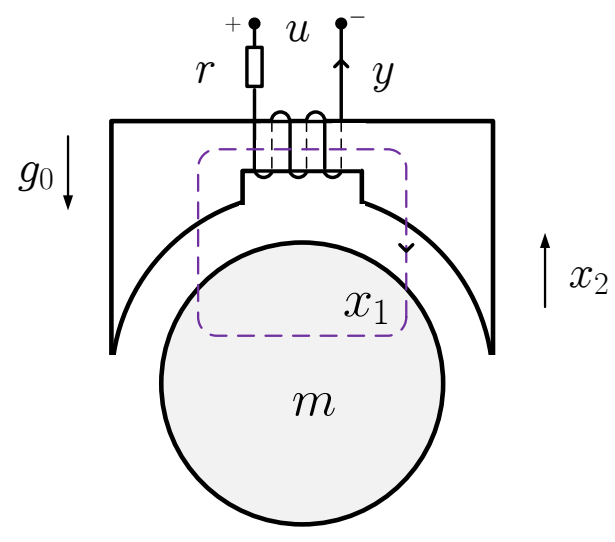

Figure 1: Levitated ball

an iron ball of mass $m$ in a vertical magnetic field as shown in Fig. 1. Assuming the flux, denoted $x_{1}$, and the current $y$ are related by

$$
x_{1}=\frac{k}{1-x_{2}} y
$$

where $k$ is a positive constant, $x_{2} \in(-\infty, 1)$ is the distance between the centre of the ball and its nominal position and taking as state vector $x=\operatorname{col}\left(x_{1}, x_{2}, m \dot{x}_{2}\right)$, the dynamics of the system is described by (1) with

$$
f(x)=\left(\begin{array}{c}
-\frac{r}{k}\left(1-x_{2}\right) x_{1} \\
\frac{x_{3}}{m} \\
\frac{1}{2 k} x_{1}^{2}-m g_{0}
\end{array}\right), g=\left(\begin{array}{l}
1 \\
0 \\
0
\end{array}\right), h(x)=\frac{1}{k}\left(1-x_{2}\right) x_{1},
$$

where $u$ is the voltage, $r$ is the inductors resistance and $g_{0}$ denotes the acceleration due to gravity - see [26] for additional details of the model.

It is clear that the observation of the state from the measurement of $y$ seems a daunting task. Our objective is to design a PEBO assuming measurable the additional signal

$$
y_{v}:=h_{v}(x)=\nabla^{\top} h(x) g=\frac{1}{k}\left(1-x_{2}\right) .
$$

The first step is to solve the immersion PDE, which in this case becomes

$$
\nabla^{\top} \phi(x)[f(x)+g u]=\beta\left(h(x), h_{v}(x), u\right) .
$$

Taking $n_{z}=1$ a feasible solution is

$$
\phi(x)=x_{3}, \beta\left(y, y_{v}, u\right)=\frac{1}{2 k}\left(\frac{y}{y_{v}}\right)^{2}-m g_{0} .
$$

The second step is the definition of the left inverse mapping, which can be chosen as 1

$$
\phi^{L}\left(x_{3}, y, y_{v}\right)=\operatorname{col}\left(\frac{y}{y_{v}}, 1-k y_{v}, x_{3}\right) .
$$

Finally, we proceed to determine the regression model and propose a parameter estimator. The dynamic part of the regression model (6)

$$
\dot{\mathfrak{z}}=\frac{1}{2 k}\left(\frac{y}{y_{v}}\right)^{2}-m g_{0}
$$

\footnotetext{
${ }^{1}$ Notice the addition of the new argument $y_{v}$ in the definitions of $\beta$ and $\phi^{L}$.
} 
However, $h$ and $h_{v}$ depend only on $x_{1}, x_{2}$ and the first two components of $\phi^{L}$ are also independent of $x_{3}$ - that we recall is $\phi(x)$ and it equals $\mathfrak{z}+\vartheta$ - consequently the composed mappings

$$
\begin{aligned}
y & =h\left(\phi^{L}\left(\mathfrak{z}+\vartheta, y, y_{v}\right)\right) \\
y_{v} & =h_{v}\left(\phi^{L}\left(\mathfrak{z}+\vartheta, y, y_{v}\right)\right)
\end{aligned}
$$

are independent of $\vartheta$, therefore, they cannot be used to pose the parameter estimation problem.

To obtain a regression model we use the dynamic relation $\dot{x}_{2}=\frac{1}{m} x_{3}$ and express it as

$$
m \dot{x}_{2}=z=(\mathfrak{z}+\vartheta) .
$$

Applying the simple LTI filter $\frac{1}{\alpha p+1}$, with $p:=\frac{d}{d t}, \alpha>0$, to this equation yields the simple relation

$$
Y=\vartheta+\epsilon_{t}
$$

where we defined the measurable signals

$$
Y:=\frac{p}{\alpha p+1} m \hat{x}_{2}-\frac{1}{\alpha p+1} \mathfrak{z},
$$

$\epsilon_{t}$ is an exponentially decaying term stemming from the filters initial conditions with $\hat{x}_{2}:=1-k y_{v}$. Thus a parameter estimator is designed as

$$
\dot{\hat{\vartheta}}=\gamma(Y-\hat{\vartheta})
$$

with $\gamma>0$ the adaptation gain. Replacing (14) in (16), and defining the parameter error $\tilde{\vartheta}:=\hat{\vartheta}-\vartheta$, we get the error equation

$$
\dot{\tilde{\vartheta}}=-\gamma \tilde{\vartheta}+\gamma \epsilon_{t},
$$

that, clearly, converges to zero exponentially fast.

The derivations above are summarised in the proposition below.

Proposition 2. Consider the system (1), (11) with additional measurable signal (12). The PEBO (13), (15), (16) with

$$
\hat{x}=\operatorname{col}\left(\frac{y}{y_{v}}, 1-k y_{v}, \mathfrak{z}+\hat{\vartheta}\right)
$$

ensures $\lim _{t \rightarrow \infty}|\hat{x}(t)-x(t)|=0$ (exponentially fast).

\section{Generation of Virtual Outputs via Signal Injection}

In [6] a novel procedure to generate alternative virtual outputs via signal injection and filtering was proposed. In words, the idea is to add to the control signal a high-frequency small amplitude periodic signal that induces an oscillation in the signal $\nabla h^{\top}(x) g(x) b$ that can be recovered filtering the plant output. Under the standing assumption that there exists a dynamic exponentially stabilising controller with inputs $y$ and $\nabla^{\top} h(x) g(x) b$ it is shown that the latter signal can be reconstructed with an accuracy $\mathcal{O}(\varepsilon)$, where $\varepsilon$ is the period of the probing signal. In [6] it is then proposed to replace the signal $\nabla^{\top} h(x) g(x) b$ by its estimate in the controller and it is shown that the trajectories of the closed-loop system converge to a neighbourhood of the equilibrium with radius $\mathcal{O}(\sqrt{\varepsilon})$.

In this section we present the extensions of the results of [6] indicated in points (i)-(iii) of Section 1. Then, we investigate how to use the virtual outputs to simplify the design of PEBO, in both critical steps of solving the PDE (2) and ensuring the left invertibility of its solution. 


\subsection{Assumption for the ideal system}

Similarly to [6], the following technical assumption regarding the global exponential stabilizability of the system (1) via dynamic feedback of the output $y$ and the virtual output $y_{v}=h_{v}(x)$ defined in (10) is needed for further developments. The qualifier "globally" can be removed at the prize of doing, throughout the paper, a far more cumbersome local analysis - we avoid these complications for the sake of clarity.

Assumption 1. There exists a dynamic controller of the form

$$
\begin{aligned}
u & =\beta\left(x_{c}, y, y_{v}\right) \\
\dot{x}_{c} & =F\left(x_{c}, y, y_{v}\right)
\end{aligned}
$$

with $\underline{\mathrm{x}}_{c} \in \mathbb{R}^{n_{x_{c}}}$, such that the zero equilibrium ${ }^{2}$ of the system

$$
\begin{aligned}
\underline{\dot{\mathrm{x}}} & =f(\underline{\mathrm{x}})+g(\underline{\mathrm{x}}) \beta\left(\underline{\mathrm{x}}_{c}, h(\underline{\mathrm{x}}), h_{v}(\underline{\mathrm{x}})\right) \\
\underline{\dot{\mathrm{x}}}_{c} & =F\left(\underline{\mathrm{x}}_{c}, h(\underline{\mathrm{x}}), h_{v}(\underline{\mathrm{x}})\right)
\end{aligned}
$$

is globally exponentially stable.

To estimate the signal $y_{v}$ we inject to the input of the plant a small amplitude, high-frequency signal

$$
s_{b}:=s\left(\frac{t}{\varepsilon}\right) b
$$

where $s(\cdot)$ is a 1-periodic, zero mean function and $b$ is a free, $m$-dimensional, constant vector that "scales" the action of the probing signal for the various input channels. It is necessary, then, to prove that if we use this estimate, denoted $\hat{y}_{v}$, in the control law (17) with the probing signal $s_{b}$ the trajectories of the closed-loop system will be "close" to those of the ideal system (18). More precisely, we need to prove that the error between the actual systems trajectories and the trajectories of the averaged ideal system is $\mathcal{O}(\varepsilon)$ - see [24] for details on this kind of analysis.

\subsection{Behaviour of the perturbed system}

To carry out the aforementioned task we first analyse the trajectories of the closed-loop system when, instead of $y_{v}$, we use in the controller a signal $\breve{y}_{v}$ verifying

$$
\breve{y}_{v}:=h_{v}(x)+\varepsilon \omega(x, t)+\varepsilon \kappa\left(x, \frac{t}{\varepsilon}\right)+\mathcal{O}\left(\varepsilon^{2}\right)
$$

where $\omega$ is a small perturbation verifying $\|\omega(\cdot, t)\|_{\infty} \leq k_{1}$, and $\kappa$ is bounded, has zero mean and is 1-periodic with respect to its second argument ${ }^{3}$ We now consider the dynamic feedback law

$$
\begin{aligned}
u & =\beta\left(x_{c}, y, \breve{y}_{v}\right)+s_{b} \\
\dot{x}_{c} & =F\left(x_{c}, y, \breve{y}_{v}\right)
\end{aligned}
$$

where $s_{b}$ is given in $(19)$, yielding the closed-loop system

$$
\begin{aligned}
\dot{x} & =f(x)+g(x) \beta\left(x_{c}, h(x), \breve{y}_{v}\right)+s\left(\frac{t}{\varepsilon}\right) g(x) b \\
\dot{x}_{c} & =F\left(x_{c}, h(x), \breve{y}_{v}\right) .
\end{aligned}
$$

\footnotetext{
${ }^{2}$ Without loss of generality it is assumed in all cases that the equilibrium of interest is the zero equilibrium.

${ }^{3}$ We will show below that it is possible to generate an estimate $\hat{y}_{v}$ verifying the condition 20 assumed for $\breve{y}_{v}$.
} 
Define a fast time scale $\tau=\frac{t}{\varepsilon}$. The averaged system of 22 is

$$
\left(\begin{array}{c}
\dot{\bar{x}} \\
\dot{\bar{x}}_{c}
\end{array}\right)=\left(\begin{array}{c}
f(\bar{x})+g(\bar{x}) \beta\left(\bar{x}_{c}, h(\bar{x}), h_{v}(\bar{x})\right) \\
F\left(\bar{x}_{c}, h(\bar{x}), h_{v}(\bar{x})\right)
\end{array}\right)+\varepsilon\left(\begin{array}{c}
g(\bar{x}) \partial_{3} \beta\left(\bar{x}_{c}, h(\bar{x}), h_{v}(\bar{x})\right) \\
\partial_{3} F\left(\bar{x}_{c}, h(\bar{x}), h_{v}(\bar{x})\right)
\end{array}\right) \omega(\bar{x}, \varepsilon \tau) .
$$

We have the following Lemma, whose proof is given in Appendix A

Lemma 1. Consider the closed-loop system (22) and its averaged behaviour (23). For all initial conditions $\left(x(0), x_{c}(0)\right) \in \mathbb{R}^{n} \times \mathbb{R}^{n_{x_{c}}}$, there exists $\varepsilon^{*}>0$, such that $\forall \varepsilon \in\left(0, \varepsilon^{*}\right]$,

$$
\left(\begin{array}{c}
x \\
x_{c}
\end{array}\right)=\left(\begin{array}{c}
\bar{x} \\
\bar{x}_{c}
\end{array}\right)+\varepsilon S\left(\frac{t}{\varepsilon}\right)\left(\begin{array}{c}
g(\bar{x}) b \\
0
\end{array}\right)+\mathcal{O}\left(\varepsilon^{2}\right)
$$

is satisfied where

$$
S(\mu):=\int_{0}^{\mu} s(\tau) \mathrm{d} \tau-\int_{0}^{1} \int_{0}^{\sigma} s(\tau) \mathrm{d} \tau \mathrm{d} \sigma .
$$

Moreover, the trajectories of the closed-loop system enter in finite time an invariant set, which is a neighbourhood of the equilibrium with radius $\mathcal{O}(\sqrt{\varepsilon})$.

\subsection{Estimation of $y_{v}$}

We are now in position to design an estimator $\widehat{y}_{v}$ for the virtual output. It is worth pointing out that in [6] the estimator is designed for the virtual output of ideal system (18), that is, ignoring the perturbation term $\Delta(\bar{\chi}, t)$ in A.2. This estimate is clearly not useful for observer design, therefore, we propose to design the estimator for the practically realisable system (22). This result is contained in the proposition below whose proof is given in Appendix B.

Proposition 3. Suppose Assumption 1 holds and consider the dynamics of the system (22). Define the estimator of the virtual output $y_{v}$ as

$$
\begin{aligned}
& \hat{y}_{v}(t)=\frac{1}{\varepsilon} \frac{\frac{1}{n \varepsilon} \int_{t-n \varepsilon}^{t}(y(\mu)-\widehat{\bar{y}}(\mu)) S\left(\frac{\mu}{\varepsilon}\right) \mathrm{d} \mu}{\frac{1}{n \varepsilon} \int_{t-n \varepsilon}^{t} S^{2}\left(\frac{\mu}{\varepsilon}\right) \mathrm{d} \mu}, \\
& \hat{\bar{y}}(t)=\frac{1}{n \varepsilon} \int_{t-n \varepsilon}^{t} y(\mu) \mathrm{d} \mu .
\end{aligned}
$$

The estimation error $\widehat{y}_{v}-y_{v}$ is $\mathcal{O}(\varepsilon)$. More precisely,

$$
\widehat{y}_{v}(t)=y_{v}(t)+\varepsilon \mathfrak{p}^{-1} \cdot \mathfrak{V}(t)+\mathcal{O}\left(\varepsilon^{2}\right) \cdot \mathbb{1}_{p},
$$

where $\mathfrak{p}$ is a constant independent of $\varepsilon$ and $\mathfrak{V}(t)$ is a bounded function whose norm is also independent of $\varepsilon$. Therefore, the estimate $\widehat{y}_{v}$ also verifies 20 with $\kappa$ equal to zero.

The following is an immediate corollary of Lemma 1 and Proposition 3.

Corollary 1. Suppose Assumption 1 holds and consider the dynamics of the system (1) in closed-loop with the control

$$
\begin{aligned}
u & =\beta\left(x_{c}, y, \hat{y}_{v}\right)+s_{b} \\
\dot{x}_{c} & =F\left(x_{c}, y, \hat{y}_{v}\right)
\end{aligned}
$$

with $\hat{y}_{v}$ generated via (25). For all initial conditions $\left(x(0), x_{c}(0)\right) \in \mathbb{R}^{n} \times \mathbb{R}^{n_{x_{c}}}$, there exists $\varepsilon^{*}>0$, such that $\forall \varepsilon \in\left(0, \varepsilon^{*}\right]$ the trajectories of the closed-loop system enter in finite time an invariant set, which is a neighbourhood of the equilibrium with radius $\mathcal{O}(\sqrt{\varepsilon})$. 


\subsection{Relaxing the exponential stabilization requirement of Assumption 1}

The following Proposition, whose proof is given in Appendix C, relaxes the exponential stabilization assumption to only asymptotic stabilization.

Proposition 4. Corollary 1 holds true even if we replace the "exponentially" qualifier in Assumption 1 by "asymptotically", with the only exception that the radius of the neighbourhood of the equilibrium to which the trajectories converge is $\mathfrak{D}(\varepsilon)$, with $\mathfrak{D}$ a class $\mathcal{K}$ function.

\section{Using $y_{v}$ to Relax the Assumptions of PEBO}

Availability of the virtual output $y_{v}$-obtained via signal injection-allows us to relax the three assumptions imposed in Proposition 1 for the design of a PEBO.

Before presenting the new assumptions we notice that in the definition of $y_{v}(10)$ we have the free $m$-dimensional vector $b$ that should be added as an additional degree of freedom in the three new assumptions below - this clarification is omitted for brevity. Also, to avoid cluttering the notation in all mappings we have used the same symbols in both sets of assumptions, only modifying their arguments.

(A1') (PDE solvability) There exist mappings

$$
\phi: \mathbb{R}^{n} \rightarrow \mathbb{R}^{n_{z}}, \quad \beta_{f}: \mathbb{R}^{p} \times \mathbb{R}^{p} \rightarrow \mathbb{R}^{n_{z}}, \quad \beta_{g}: \mathbb{R}^{p} \times \mathbb{R}^{p} \rightarrow \mathbb{R}^{n_{z} \times m}
$$

with $n_{z} \geq n$ satisfying the PDE

$$
\begin{aligned}
& f^{\top}(x) \nabla \phi(x)=\beta_{f}^{\top}\left(h(x), h_{v}(x)\right) \\
& g^{\top}(x) \nabla \phi(x)=\beta_{g}^{\top}\left(h(x), h_{v}(x)\right)
\end{aligned}
$$

(A2') (Left invertibility) There exists a mapping $\phi^{L}: \mathbb{R}^{n_{z}} \times \mathbb{R}^{p} \times \mathbb{R}^{p} \rightarrow \mathbb{R}^{n}$ such that

$$
\phi^{L}\left(\phi(x), h(x), h_{v}(x)\right)=x .
$$

(A3') (Consistent identification) There exists mappings

$$
M: \mathbb{R}^{n_{\zeta}} \times \mathbb{R}^{n_{z}} \times \mathbb{R}^{p} \times \mathbb{R}^{p} \times \mathbb{R}^{m} \rightarrow \mathbb{R}^{n_{\zeta}}, N: \mathbb{R}^{n_{\zeta}} \times \mathbb{R}^{n_{z}} \times \mathbb{R}^{p} \times \mathbb{R}^{p} \times \mathbb{R}^{m} \rightarrow \mathbb{R}^{n_{z}},
$$

with $n_{\zeta} \in \mathbb{N}_{+}$such that the parameter estimator

$$
\begin{aligned}
\dot{\zeta} & =M\left(\zeta, \mathfrak{z}, y, y_{v}, u\right) \\
\hat{\vartheta} & =N\left(\zeta, \mathfrak{z}, y, y_{v}, u\right)
\end{aligned}
$$

generates a consistent estimate for the nonlinear regression model

$$
\begin{aligned}
\dot{\mathfrak{z}} & =\beta\left(y, y_{v}, u\right) \\
y & =h\left(\phi^{L}\left(\mathfrak{z}+\vartheta, y, y_{v}\right)\right),
\end{aligned}
$$

where

$$
\beta\left(y, y_{v}, u\right):=\beta_{f}\left(y, y_{v}\right)+\beta_{g}\left(y, y_{v}\right) u .
$$

Notice that - exploiting the affine dependence on $u$ of the PDE (2) - we have split it into two parts in Assumption ( $\left.\mathrm{A} 1^{\prime}\right)$. It is interesting to observe also that the addition of the virtual output considerably simplifies the solution of the second PDE in (28). Indeed, we recall that it can be written in the form

$$
\nabla^{\top} \phi(x) g(x)=\beta_{g}\left(h(x), \nabla^{\top} h(x) g(x) b\right)
$$

where we underscore the presence of the matrix $g(x)$ on both sides of the equation. Moreover, the free vector $b$ can be selected to simplify the solution, a fact that is illustrated in the example of Subsection 7.2 


\section{A procedure to solve the PDE (28)}

In this section we show that the solution of the key PDE (28) can be translated into the solvability of a set of (nonlinear) algebraic equations and an, easily verifiable, integrability condition - both steps disposing of several free mappings.

Decomposing the vectors $\phi(x)$ and $\beta_{f}\left(h(x), h_{v}(x)\right)$ into its $n_{z}$ components $\phi_{i}(x)$ and $\beta_{f, i}\left(h(x), h_{v}(x)\right)$ the first equation of $(28)$ can be equivalently written as

$$
f^{\top}(x) \nabla \phi_{i}(x)=\beta_{f, i}\left(h(x), h_{v}(x)\right), i=1, \ldots, n_{z} .
$$

The general solution of these equations is of the form

$$
\nabla \phi_{i}(x)=\left(f^{\top}(x)\right)^{\dagger} \beta_{f, i}\left(h(x), h_{v}(x)\right)+\left[I-\left(f^{\top}(x)\right)^{\dagger} f^{\top}(x)\right] \mathcal{P}(x)=: \Phi_{i}^{f}(x)
$$

with $\mathcal{P}: \mathbb{R}^{n} \rightarrow \mathbb{R}^{n}$ is a free mapping and we recall that $(\cdot)^{\dagger}$ stands for the pseudo-inverse.

Similarly, denoting with $\beta_{g, i}^{\top}\left(h(x), h_{v}(x)\right)$ the $i$-th row of $\beta_{g}\left(h(x), h_{v}(x)\right)$, the second equation of (28) can be written element-by-element as

$$
g^{\top}(x) \nabla \phi_{i}(x)=\beta_{g, i}^{\top}\left(h(x), h_{v}(x)\right), i=1, \ldots, n_{z}
$$

whose solution is

$$
\nabla \phi_{i}(x)=\left(g^{\top}(x)\right)^{\dagger} \beta_{g, i}^{\top}\left(h(x), h_{v}(x)\right)+\left[I-\left(g^{\top}(x)\right)^{\dagger} g^{\top}(x)\right] \mathcal{Q}(x)=: \Phi_{i}^{g}(x)
$$

with $\mathcal{Q}: \mathbb{R}^{n} \rightarrow \mathbb{R}^{n}$ a free mapping.

For the right hand sides of (36) and (38) to qualify as solutions of the PDE it is necessary to impose the following two additional constraints.

(C1) Both expressions are equal, that is,

$$
\Phi_{i}^{f}(x)=\Phi_{i}^{g}(x), i=1, \ldots, n_{z} .
$$

(C2) The mappings $\Phi_{i}^{f}(x)$ are gradient vector fields $4^{4}$, that is

$$
\nabla \Phi_{i}^{f}(x)=\left(\nabla \Phi_{i}^{f}(x)\right)^{\top}, i=1, \ldots, n_{z} .
$$

The derivations above established the following proposition.

Proposition 5. For all mappings

$$
\beta_{f, i}: \mathbb{R}^{p} \times \mathbb{R}^{p} \rightarrow \mathbb{R}, \beta_{g, i}: \mathbb{R}^{p} \times \mathbb{R}^{p} \rightarrow \mathbb{R}^{m}, \mathcal{P}: \mathbb{R}^{n} \rightarrow \mathbb{R}^{n}, \mathcal{Q}: \mathbb{R}^{n} \rightarrow \mathbb{R}^{n}
$$

verifying (39) and (40), where $\Phi_{i}^{f}(x)$ and $\Phi_{i}^{g}(x)$ are defined in (36) and (38), respectively, the mapping

$$
\phi(x)=\int_{0}^{1}\left(\Phi^{f}(s x)\right)^{\top} x d s
$$

is a solution of the PDE 28.

\section{Examples}

In this section we give several examples that illustrate the use of the various propositions above.

\footnotetext{
${ }^{4}$ This condition stems from the well-known Poincare's Lemma.
} 
7.1. Solving the PDE for an indistinguishable system

Consider the system

$$
\begin{aligned}
\dot{x}_{1} & =x_{2}+u \\
\dot{x}_{2} & =x_{1} \\
y & =x_{1}^{2} .
\end{aligned}
$$

Clearly, $y$ cannot help distinguish between $\pm x_{1}$. This system is of instantaneous indistinguishability [5]. Some simple computations show that $h_{v}(x)=2 x_{1}$ and, using this virtual output, the PDE (28) admits the solution

$$
\phi(x)=x_{2}, \beta_{f}\left(h(x), h_{v}(x)\right)=\frac{1}{2} h_{v}(x), \beta_{g}\left(h(x), h_{v}(x)\right)=0 .
$$

\subsection{Solving the PDE for a port-Hamiltonian system}

Consider the port-Hamiltonian system [27]

$$
\begin{aligned}
\dot{x} & =F(x) \nabla H(x)+g(x) u \\
y & =x_{a}
\end{aligned}
$$

with $x:=\operatorname{col}\left(x_{u}, x_{a}\right) \in \mathbb{R}^{n}, x_{u} \in \mathbb{R}^{n-m}, x_{a} \in \mathbb{R}^{m}, u \in \mathbb{R}^{m}, H: \mathbb{R}^{n} \rightarrow \mathbb{R}$ the systems Hamiltonian, $F: \mathbb{R}^{n} \rightarrow \mathbb{R}^{n \times n}$ verifies

$$
F(x)+F^{\top}(x) \leq 0
$$

and

$$
g(x)=\left(\begin{array}{c}
0 \\
g_{a}(x)
\end{array}\right)
$$

The virtual output is, clearly, $y_{v}=g_{a}(x) b$. To streamline the next proposition, and consistent with the definition of $x$ lets partition $F(x)$ as

$$
F(x)=\left(\begin{array}{cc}
F_{u u}(x) & F_{u a}(x) \\
F_{a u}(x) & F_{a a}(x)
\end{array}\right)
$$

Proposition 6. If there exist constant matrices $b \in \mathbb{R}^{m}, P_{u} \in \mathbb{R}^{n \times(n-m)}, P_{a} \in \mathbb{R}^{n \times m}$ and a mapping $\mathcal{T}: \mathbb{R}^{m} \times \mathbb{R}^{m \times m} \rightarrow \mathbb{R}^{n}$ satisfying the algebraic equation

$$
\mathcal{T}\left(x_{a}, g_{a}(x) b\right)=\left(P_{u} F_{u u}(x)+P_{a} F_{a u}(x)\right) \nabla_{x_{u}} H(x)+\left(P_{u} F_{u a}(x)+P_{a} F_{a a}(x)\right) \nabla_{x_{a}} H(x),
$$

then the PDE (28) admits a solution

$$
\begin{aligned}
\phi(x) & =P_{u} x_{u}+P_{a} x_{a} \\
\beta_{f}\left(x_{a}, g_{a}(x) b, u\right) & =\mathcal{T}\left(x_{a}, g_{a}(x) b\right) \\
\beta_{g}\left(x_{a}, g_{a}(x) b, u\right) & =P_{a} g_{a}(x) .
\end{aligned}
$$

Proof 2. The proof is established using the virtual output and differentiating $\phi(x)$. 


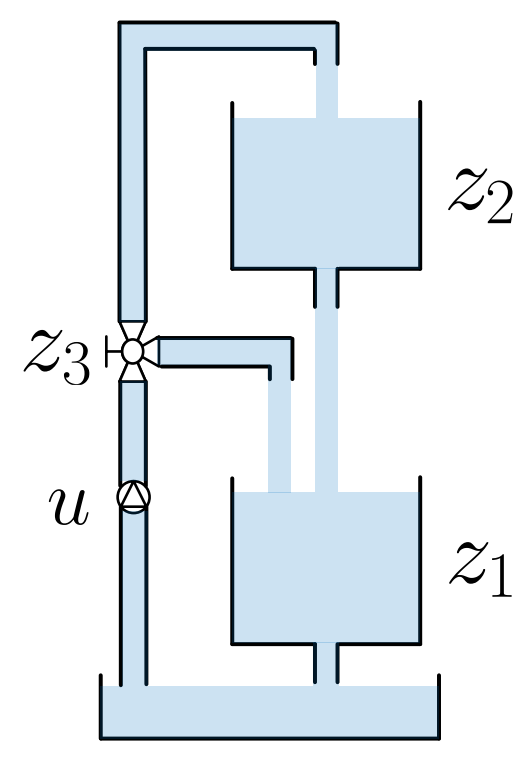

Figure 2: Two tank system

\subsection{Application to a two-tank system}

In this subsection, a two-tank system is utilized to verify the procedure to solve the PEBO PDE, proposed in Section 6. Consider the two-tank system depicted in Fig 2, As shown in [7] the dynamics is described by (1) with

$$
\dot{z}=\left(\begin{array}{c}
-\frac{a_{1}}{A_{1}} \sqrt{z_{1}}+\frac{a_{2}}{A_{1}} \sqrt{z_{2}} \\
-\frac{a_{2}}{A_{2}} \sqrt{z_{2}} \\
0
\end{array}\right)+\left(\begin{array}{c}
\frac{1}{A_{1}} z_{3} \\
\frac{1}{A_{2}}\left(1-z_{3}\right) \\
0
\end{array}\right) u, y=z_{1}
$$

where the states $z_{1}, z_{2} \in \mathbb{R}_{+}$represent the water level in the lower and upper tank, the constant $z_{3} \in[0,1]$ is the unknown valve parameter, with the extreme values corresponding to fully open and closed, and all the parameters are positive constants.

The virtual output is $h_{v}(z)=\frac{z_{3}}{A_{1}}$. To simplify the dynamics we introduce the change of coordinates

$$
x=\left(\begin{array}{c}
A_{1} z_{1}+A_{2} z_{2} \\
z_{2} \\
z_{3}
\end{array}\right)
$$

for which the dynamics are given by

$$
\begin{aligned}
\dot{x}_{1} & =-a_{1} \sqrt{y}+u \\
\dot{x}_{2} & =\frac{a_{2}}{A_{2}} \sqrt{x_{2}}+\frac{1}{A_{2}}\left(1-x_{3}\right) u \\
\dot{x}_{3} & =0,
\end{aligned}
$$

where, with some abuse of notation, we have written the first term in the right hand side of the $x_{1}$ dynamics in terms of $y\left(=z_{1}\right)$. We have the following proposition.

Proposition 7. A family of solutions of the PDE (28) for the two-tank system dynamics are given by

$$
\begin{aligned}
\phi(x) & =\gamma_{1}\left(x_{3}\right) x_{1}+\gamma_{2}\left(x_{3}\right) \\
\beta\left(y, y_{v}\right) & =\gamma_{1}\left(A_{1} y_{v}\right)\left(-a_{1} \sqrt{y}+u\right) .
\end{aligned}
$$

with $\gamma_{1}, \gamma_{2}: \mathbb{R}_{+} \rightarrow \mathbb{R}$ free functions. 
Proof 3. The PDEs (28) take the form

$$
\begin{aligned}
\nabla_{x_{1}} \phi(x)\left(-a_{1} \sqrt{y}\right)+\nabla_{x_{2}} \phi(x)\left(\frac{a_{2}}{A_{2}} \sqrt{x_{2}}\right) & =\beta_{f}\left(y, y_{v}\right) \\
\nabla_{x_{1}} \phi(x)+\nabla_{x_{2}} \phi(x)\left(\frac{1}{A_{2}}\left(1-x_{3}\right)\right) & =\beta_{g}\left(y, y_{v}\right) .
\end{aligned}
$$

From the equations above we conclude that $\phi$ cannot depend on $x_{2}$ and its dependence with respect to $x_{1}$ should be linear. On the other hand, it can depend on $x_{3}$ via arbitrary functions. Replacing this facts in the equations above denied the functions $\beta_{f}\left(y, y_{v}\right)$ and $\beta_{g}\left(y, y_{v}\right)$.

The obtained solution is left invertible with

$$
\begin{aligned}
& x_{1}=\frac{\phi(x)-\gamma_{2}\left(A_{1} y_{v}\right)}{\gamma_{1}\left(A_{1} y_{v}\right)} \\
& x_{2}=\frac{\phi(x)-\gamma_{2}\left(A_{1} y_{v}\right)}{\gamma_{1}\left(A_{1} y_{v}\right) A_{2}}-\frac{A_{1} y}{A_{2}} \\
& x_{3}=A_{1} y_{v} .
\end{aligned}
$$

Clearly, the PDE solution is left invertible when $\gamma_{1} \neq 0$ for all $x$.

\subsection{Simulation results of the magnetic levitation system (cont'd)}

In this subsection we present some simulation results of the magnetic levitation system studied in Section 3. The system is placed in closed-loop with the full state-feedback asymptotically stabilising passivity-based controller proposed in [23], where the states are replaced by the estimates generated via $\mathrm{PEBO}$ with signal injection.

The control law is given by

$$
u=r y+K_{P}\left(\frac{1}{a}\left(\hat{x}_{1}-\sqrt{2 k m g_{0}}\right)+\hat{x}_{2}-x_{2 *}\right)-\frac{\alpha}{m} \hat{x}_{3}-\frac{r}{a}\left(\frac{1}{2 k} \hat{x}_{1}^{2}-m g_{0}\right)+s\left(\frac{t}{\varepsilon}\right)
$$

where $x_{2 *} \in(-\infty, 1)$ is the desired position of the ball and the PEBO with virtual output is generated by

$$
\begin{aligned}
\dot{\mathfrak{z}} & =\frac{1}{2 k}\left(\frac{y}{\hat{y}_{v}}\right)^{2}-m g_{0} \\
Y & =\frac{p}{\alpha p+1} m x_{2}-\frac{1}{\alpha p+1} \mathfrak{z} \\
\dot{\hat{\vartheta}} & =\gamma(Y-\hat{\vartheta}) \\
\hat{x} & =\operatorname{col}\left(\frac{y}{\hat{y}_{v}}, 1-k \hat{y}_{v}, \mathfrak{z}+\hat{\vartheta}\right)
\end{aligned}
$$

with $\hat{y}_{v}$ obtained from (25).

The simulation scenario was as follows: all parameters of the system are set equal to one, the controller gains were $K_{P}=12, a=1, b=1, \alpha=4.5$ and $\gamma=1$. The desired position is $x_{2 *}=0.5$, the initial values are given as $x(0)=\operatorname{col}(0,0,0), \mathfrak{z}(0)=1, \hat{\vartheta}(0)=0$ and the initial conditions of the filters set to zero. A $10^{-3}$-periodic sine function is utilized as signal injection, which corresponds to $\varepsilon=0.001$.

The simulation results are given in Fig. 3, which shows the evolution of all signals of interest. The plot of the virtual output and its estimate verify the efficiency of the estimator (25), since consistent with the prediction of the theory, the estimation error is around $\mathcal{O}(\varepsilon)$. The plot of dynamic extension $\mathfrak{z}$ and $x_{3}$ clearly illustrate that, after a certain transient due to the initial error of $\widehat{y}_{v}$, they verify the relation $\mathfrak{z}(t)=x_{3}(t)+\vartheta$, with the constant deviation being effectively estimated as seen from the plot of $\hat{x}_{3}$. The history of the control input $u$, is shown in the last figure with and without signal injection term $s_{b}$, highlighting that the high frequency signal added to the input is effectively "filtered" by the system dynamics rendering it, essentially, unobservable. 

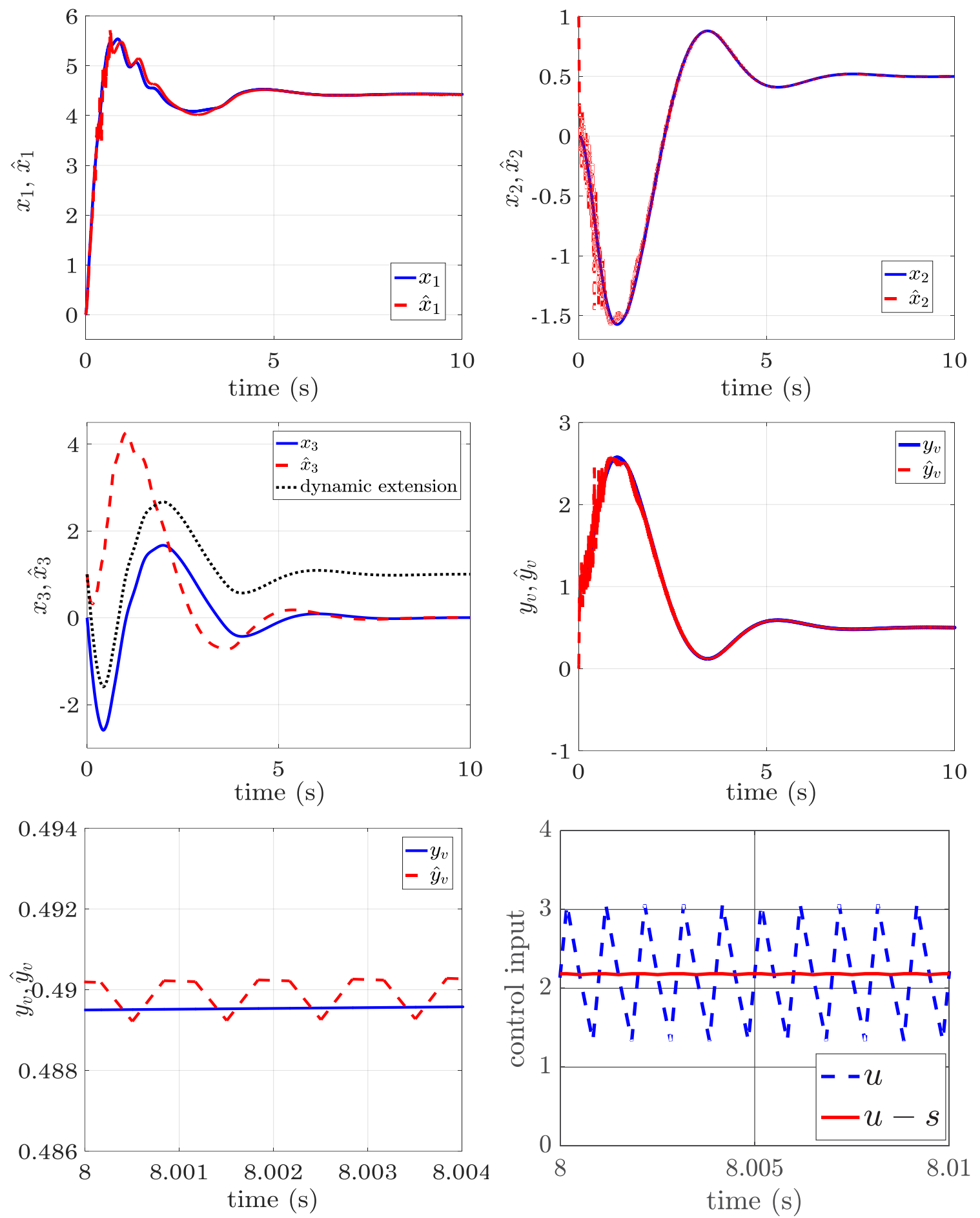

Figure 3: Simulation results of the levitated ball 


\section{Conclusions and Future Research}

We have investigated how to relax the conditions for the design of the PEBO for nonlinear systems reported in [22]. The first modification is the introduction of the signal injection proposed in [6] to generate new "virtual outputs" that are incorporated in the PEBO design. To carry out this objective it was necessary to provide some extensions to the work of [6]. Although not discussed in this paper it is clear that the addition of these new outputs also relaxes the left invertibility and parameter identifiability conditions of $\mathrm{PEBO}$ - these features are illustrated with some representative examples including a novel sensorless controller for a magnetic levitation system. The second main modification is to translate the problem of solving the key PDE into the solution of a set of nonlinear algebraic equations and an integrability condition, with both steps including some free parameters. This new procedure is similar to the so-called "algebraic solution" of interconnection and damping assignment passivity-based controllers proposed in [9], see also [21], which has proven successful in some applications where solving the PDE is a hard task.

Some problems that need to be further studied include the following,

- Identify physical systems satisfying the relaxed requirements of PEBO, especially for electromechanical systems to obtain sensorless controllers.

- Study the sensitivity of PEBO to measurement noise, this issue is particularly critical since PEBO sometimes involves the open-loop integration of signals.

- Investigate the conditions under which PEBO yields a "tractable" parameter estimation problem beyond the linear dependence case studied in [22]-considering, for instance, monotonicity or convexity/concavity dependences.

- The connection between PEBO, KKL and immersion and invariance observers is currently under investigation and we expect to report soon a unifying framework for all of them.

\section{Acknowledgement}

This paper is partly supported by the National Natural Science Foundation of China (61473183, U1509211), the Government of Russian Federation (074U01, GOSZADANIE 2014/190 (project 2118), and the Ministry of Education and Science of Russian Federation (14.Z50.31.0031). The support

provided by China Scholarship Council during a visit of the first author to L2S, CNRS-CentraleSupélec is acknowledged.

\section{References}

[1] M. Alamir, Optimization based non-linear observers revisited, Int. J. of Control., vol. 72, pp. 1204-1217, 1999.

[2] V. Andrieu and L. Praly, On the existence of a Kazantzis-Kravaris/Luenberger observer, SIAM J. on Control and Optim., vol. 45, pp. 432-456, 2006.

[3] M. Arcak and P. Kokotović, Nonlinear observers: a circle criterion design and robustness analysis, Automatica, vol. 37, pp. 1923-1930, 2001.

[4] A. Astolfi, D. Karagiannis and R. Ortega, Nonlinear and Adaptive Control with Applications, Springer, London, 2008.

[5] G. Besançon, Nonlinear Observers and Applications, Springer, 2007.

[6] P. Combes, A. K. Jebai, F. Malrait, P. Martin and P. Rouchon, Adding virtual measurements by signal injection, 2016 American Control Conference, Boston, USA, July 6-8, pp. 999-1005, 2016. 
[7] E. García-Canseco, D. Jeltsema, R. Ortega and J. M.A. Scherpen, Power-based control of physical systems, Automatica, vol. 46, pp. 127-132, 2010.

[8] F. Esfandiari and H. K. Khalil, Output feedback stabilization of fully linearizable systems, Int. J. of Control, vol. 56, pp. 1007-1037, 1992.

[9] K. Fujimoto and T. Sugie, Canonical transformation and stabilization of generalized Hamiltonian systems, Systems \& Control Letters, vol. 42, pp. 217-227, 2001.

[10] J. P. Gauthier and I. Kupka, Deterministic Observation Theory and Applications, Cambridge University Press, 2001.

[11] J. P. Gauthier, H. Hammouri and S. Othman, A simple observer for nonlinear systems applications to bioreactors, IEEE Trans. on Automatic Control, vol. 37, pp. 875-880, 1992.

[12] G. Hernandez-Gonzalez and R. Iravani, Current injection for active islanding detection of electronically-interfaced distributed resources. IEEE Trans. on Power Delivery, Vol. 21, No. 3, pp. 1698-1705, 2006.

[13] J. Holtz, Sensorless control of induction motor drives, Proc. of the IEEE, vol. 90, pp. 1359-1394, 2002.

[14] D. Karagiannis, D. Carnevale and A. Astolfi, Invariant manifold based reduced-order observer design for nonlinear systems, IEEE Trans. on Automatic Control, vol. 53, pp. 2602-2614, 2008.

[15] N. Kazantzis and C. Kravaris, Nonlinear observer design using Lyapunov's auxiliary theorem, Systems 8 Control Letters, vol. 34, pp. 241-247, 1998.

[16] H. K. Khalil, Noninear Systems, Prentice-Hall, New Jersey, 2002.

[17] G. Kreisselmeier and R. Engel, Nonlinear observers for autonomous Lipschitz continuous systems, IEEE Trans. on Automatic Control, vol. 48, pp. 451-464, 2003.

[18] A. J. Krener and A. Isidori, Linearization by output injection and nonlinear observers, Systems 63 Control Letters, vol. 3, pp. 47-52, 1983.

[19] D. G. Luenberger, Observers for multivariable systems, IEEE Trans. on Automatic Control, vol. 11, No. 2, pp. 190-197, 1966.

[20] P. T. Kabamba, S. M. Meerkov and E-K. Poh, Pole placement capabilities of vibrational control, IEEE Trans. on Automatic Control, vol. 43, pp. 1256-1261, 1998.

[21] R. Ortega and E. G. Canseco, Interconnection and damping assignment passivity-based control: A survey, European J. of Control, vol. 10, pp. 432-450, 2004.

[22] R. Ortega, A. Bobtsov, A. Pyrkin and S. Aranovskiy, A parameter estimation approach to state observation of nonlinear systems, Systems \& Control Letters, vol. 85, pp. 84-94, 2015.

[23] R. Ortega, A. J. van der Schaft, I. Mareels and B. Maschke, Putting energy back in control, IEEE Control Systems Magazine, vol. 21, pp. 18-33, 2001.

[24] J. A. Sanders, F. Verhulst, J. Murdock, Averaging Methods in Nonlinear Dynamical Systems, Springer, New York, 2007.

[25] S. Sastry and M. Bodson, Adaptive Control: Stability, Convergence and Robustness, Prentice Hall, Englewood Cliffs, NJ, 1989. 
[26] M. Torres and R. Ortega, Feedback linearization, integrator backstepping and passivity-based controller design: A comparison example, in Perspectives in Control: Theory and Application, ed. D. Normand-Cyrot, Springer-Verlag, Londres, 1998.

[27] A. J. van der Schaft, L2-gain and Passivity Techniques in Nonlinear Control, Springer, 2017.

\section{Appendix A. Proof of Lemma 1}

Define $\chi:=\operatorname{col}\left(x, x_{c}\right)$. In the fast time scale $\tau=\frac{t}{\varepsilon}$, the dynamics become

$$
\frac{\mathrm{d} \chi}{\mathrm{d} \tau}=\varepsilon\left(F_{1}^{(1)}(\chi)+F_{1}^{(2)}(\chi) s(\tau)\right)+\varepsilon^{2} F_{2}(\chi, \tau)+\mathcal{O}\left(\varepsilon^{3}\right)
$$

where

$$
\begin{aligned}
F_{1}^{(1)} & :=\left(\begin{array}{c}
f(x)+g(x) \beta\left(x_{c}, h(x), h_{v}(x)\right) \\
F\left(x_{c}, h(x), h_{v}(x)\right)
\end{array}\right), \\
F_{1}^{(2)} & :=\operatorname{col}(g(x) b, 0), \\
F_{2} & :=\left(\begin{array}{c}
g(x) \partial_{3} \beta\left(x_{c}, y, \breve{y}_{v}\right) \cdot\left(\kappa\left(x, \frac{t}{\varepsilon}\right)+\omega(x, t)\right) \\
\partial_{3} F\left(x_{c}, y, \breve{y}_{v}\right)\left(\kappa\left(x, \frac{t}{\varepsilon}\right)+\omega(x, t)\right)
\end{array}\right) .
\end{aligned}
$$

Following [6], we use the second-order averaging approach to obtain the averaged system of (22). Considering the periodic terms $F_{1}^{(2)}(\chi) s(\tau)$ and $\kappa(\tau)$, the averaged system in time scale $t$ is 5

$$
\dot{\bar{\chi}}=\left(\begin{array}{c}
f(\bar{x})+g(\bar{x}) \beta\left(\overline{x_{c}}, h(\bar{x}), h_{v}(\bar{x})\right) \\
F\left(\overline{x_{c}}, h(\bar{x}), h_{v}(\bar{x})\right)
\end{array}\right)+\varepsilon \Delta(\bar{\chi}, t)
$$

where

$$
\begin{aligned}
\Delta(\bar{\chi}, \varepsilon \tau) & :=\operatorname{col}\left(\Delta_{1}, \Delta_{2}\right) \\
& =\left(\begin{array}{c}
g(\bar{x}) \cdot \partial_{3} \beta\left(\bar{x}_{c}, y, \breve{y}_{v}\right) \omega(\bar{x}, \varepsilon \tau) \\
\partial_{3} F\left(\bar{x}_{c}, y, \breve{y}_{v}\right) \omega(\bar{x}, \varepsilon \tau)
\end{array}\right),
\end{aligned}
$$

which is a perturbed system of the nominal system (18). In the interval $[t, t+\mathcal{O}(1 / \varepsilon))$ we have

$$
\left(\begin{array}{c}
x \\
x_{c}
\end{array}\right)=\left(\begin{array}{c}
\bar{x} \\
\overline{x_{c}}
\end{array}\right)+\varepsilon S\left(\frac{t}{\varepsilon}\right)\left(\begin{array}{c}
g(\bar{x}) b \\
0
\end{array}\right)+\mathcal{O}\left(\varepsilon^{2}\right) .
$$

Thus, noticing that

$$
\nabla^{\top} h(\mathfrak{x}) g(\mathfrak{x}) b S\left(\frac{t}{\varepsilon}\right)=h_{v}(x(t)) S\left(\frac{t}{\varepsilon}\right)+\mathcal{O}(\varepsilon),
$$

with $\mathfrak{x}:=x(t)-\varepsilon g(x) b S\left(\frac{t}{\varepsilon}\right)+\mathcal{O}\left(\varepsilon^{2}\right)$, we have

$$
y=h(\bar{x})+\varepsilon h_{v}(x) S+\mathcal{O}\left(\varepsilon^{2}\right)
$$

Let us proceed now with the perturbation analysis of the averaged system. According to Assumption 1 , for $\left(\bar{x}, \bar{x}_{c}\right) \in \mathcal{D} \subset \mathbb{R}^{n} \times \mathbb{R}^{n_{x_{c}}}$, where $\mathcal{D}$ is any compact set covering the equilibrium, there exist nonnegative constants $k_{i}(i=2, \ldots, 5)$ such that $\left|\Delta_{1}\right| \leq k_{2}|\bar{\chi}|+k_{3}$ and $\left|\Delta_{2}\right| \leq k_{4}|\bar{\chi}|+k_{5}$. Define

$$
\mathbf{F}\left(\underline{\mathrm{x}}, \underline{\mathrm{x}}_{c}\right):=\left(\begin{array}{c}
f(\underline{\mathrm{x}})+g(\underline{\mathrm{x}}) \beta\left(\underline{\mathrm{x}}_{c}, h(\underline{\mathrm{x}}), h_{v}(\underline{\mathrm{x}})\right) \\
F\left(\underline{\mathrm{x}}_{c}, h(\underline{\mathrm{x}}), h_{v}(\underline{\mathrm{x}})\right)
\end{array}\right)
$$

\footnotetext{
${ }^{5}\left({ }^{-}\right)$denotes the states in the averaged system.
} 
According to the converse Lyapunov theorem of exponential stability [16], there exist a smooth and positive definite function $V(\underline{\chi})$ and constants $c_{i} \in \mathbb{R}_{+}(i=1, \ldots, 3)$ for $\left(\underline{\mathrm{x}}, \underline{\mathrm{x}}_{c}\right) \in \mathbb{R}^{n} \times \mathbb{R}^{n_{x_{c}}}$ such that

$$
\begin{aligned}
& c_{1}|\underline{\chi}|^{2} \leq V(\underline{\chi}) \leq c_{2}|\underline{\chi}|^{2}, \\
& \nabla^{\top} V(\underline{\chi}) \mathbf{F}\left(\underline{\mathrm{x}}, \underline{\mathrm{x}}_{c}\right) \leq-c_{3}|\underline{\chi}|^{2} .
\end{aligned}
$$

For any $\mathcal{D}$ there exists a constant $c_{4}$ such that $c_{4}:=\sup _{\underline{\chi} \in \mathcal{D}}|\nabla V(\underline{\chi})|$. For the averaged $\left(\bar{x}, \bar{x}_{c}\right)$-system A.2, we have

$$
\dot{V}\left(\bar{x}, \bar{x}_{c}\right) \leq-c_{5}|\bar{\chi}|^{2}+\varepsilon c_{6}
$$

where $c_{5}(\varepsilon)=c_{3}-\varepsilon \max \left(k_{2}, k_{4}\right) / 2$ and $c_{6}=\max \left(k_{2}, k_{4}\right) c_{4}^{2} / 2+c_{4} \max \left(k_{3}, k_{5}\right)$.

There exists a constant $\varepsilon^{(1)} \in(0,1]$ such that $c_{5}\left(\varepsilon^{(1)}\right)>0$, and define $\underline{c}_{5}=c_{5}\left(\varepsilon^{(1)}\right)$. It yields that for $\ell \in(0,1)$,

$$
\dot{V}\left(\bar{x}, \bar{x}_{c}\right) \leq-(1-\ell) \underline{c}_{5}|\bar{\chi}|^{2}, \quad \forall \bar{\chi} \notin \Omega^{(1)}
$$

where

$$
\Omega^{(1)}:=\left\{\bar{\chi} \in \mathbb{R}^{n+n_{x_{c}}}|| \bar{\chi} \mid \leq \sqrt{\varepsilon \frac{c_{6}}{\ell_{c_{5}}}}\right\} .
$$

There exists $\varepsilon^{(2)} \in(0,1]$ such that $\Omega^{(1)} \subset \mathcal{D}$, thus selecting $\varepsilon^{*}$ as $\varepsilon^{*}=\min \left(\varepsilon^{(1)}, \varepsilon^{(2)}\right)$. For $\varepsilon \in\left(0, \varepsilon^{*}\right]$, the state of averaged system will exponentially converge into an invariant set with radius $\mathcal{O}(\sqrt{\varepsilon})$.

The correlation A.3 and Proposition 3 holds in the interval $[0, \mathcal{O}(1 / \varepsilon))$. Suppose an interval $\left[0, T_{1}^{*}\right] \subset[0, \mathcal{O}(1 / \varepsilon))$, where $T_{1}^{*}:=k_{1}^{*} / \varepsilon$ and $k_{1}^{*} \in \mathbb{R}_{+}$. According to the second-order average theorem, there is another averaged system, namely Ave-2-system 6 , having the same dynamical equation as A.2 with the initial value as

$$
\bar{\chi}^{(2)}\left(T_{1}^{*}\right)=\bar{\chi}\left(T_{1}^{*}\right),
$$

and its flow is defined as $\bar{\chi}^{(2)}\left(t ; T_{1}^{*}, \chi\left(T_{1}^{*}\right)\right)$. It should be pointed out that the Ave-2-system is asymptotically stable w.r.t. an invariant set. Thus there always exists a moment $T_{2}^{*}:=T_{1}^{*}+k_{2}^{*} / \varepsilon$ such that

$$
\left(\begin{array}{c}
x \\
x_{c}
\end{array}\right)=\left(\begin{array}{c}
\bar{x}^{(2)} \\
\bar{x}_{c}^{(2)}
\end{array}\right)+\varepsilon S\left(\frac{t}{\varepsilon}\right)\left(\begin{array}{c}
g\left(\bar{x}^{(2)}\right) \\
0
\end{array}\right)+\mathcal{O}\left(\varepsilon^{2}\right) .
$$

holds in $\left[T_{1}^{*}, T_{2}^{*}\right]$, and

$$
y(t)=h\left(\bar{x}^{(2)}(t)\right)+\varepsilon h_{v}\left(\bar{x}^{(2)}(t)\right) S\left(\frac{t}{\varepsilon}\right)+\mathcal{O}\left(\varepsilon^{2}\right)
$$

Eq. (26) still holds in the interval $\left[T_{1}^{*}, T_{2}^{*}\right]$.

Iterating the above procedure to construct the Ave- $i$-system $(i=3, \ldots, n, \ldots)$ with updated initial states

$$
\bar{\chi}^{(i)}\left(T_{i-1}^{*}\right)=\bar{\chi}^{(i-1)}\left(T_{i-1}^{*}\right),
$$

which do not need to be known in the design, with

$$
T_{i}^{*}=\sum_{j=1}^{i} \frac{k_{i}^{*}}{\varepsilon},
$$

it yields

$$
\left(\begin{array}{c}
x \\
x_{c}
\end{array}\right)=\left(\begin{array}{c}
\bar{x}^{(i)} \\
\bar{x}_{c}^{(i)}
\end{array}\right)+\varepsilon S\left(\frac{t}{\varepsilon}\right)\left(\begin{array}{c}
g\left(\bar{x}^{(i)}\right) \\
0
\end{array}\right)+\mathcal{O}\left(\varepsilon^{2}\right) .
$$

We obtain that the virtual output estimator in Proposition 3 still works with the accuracy as 26 in $\left[T_{i}^{*}, T_{i+1}^{*}\right]$. Notice that $\mathcal{O}(\sqrt{\varepsilon})+\mathcal{O}(\varepsilon)=\mathcal{O}(\sqrt{\varepsilon})$, for $\varepsilon \in(0,1)$. It completes the proof.

\footnotetext{
${ }^{6}$ The superscript $(\cdot)^{(i)}$ denotes the state in the Ave- $i$-system.
} 


\section{Appendix B. Proof of Proposition 3}

Caveat: We should bear in mind that we do not know whether $\breve{y}_{v}=\hat{y}_{v}$ verifies (20). Thus, the result in Lemma 1 cannot be utilized in the proof. However, even if $\hat{y}_{v}$ is not in the form $(20)$, following the similar analysis method in Appendix A, there exists an averaged system which keeps the relationship (A.4) when probing signals. This is the key identity in the following proof, i.e., (B.1). To avoid cluttering the notations, whenever clear from context, we still use '( $\bar{\cdot})$ ' to denote the states of this new averaged system.

The proof mimics the derivations of [6]. Assumptions 1 guarantees that $\dot{\bar{y}}, \ddot{\bar{y}}$ and $\dot{\bar{y}}_{v}$ exist for the averaged system (A.2). We can, thus, obtain a Taylor series expansion of third order and second order of $\bar{y}$ and $\bar{y}_{v}$, respectively. That is,

$$
\begin{aligned}
& \bar{y}(t-\tau)=\bar{y}(t)-\tau \dot{\bar{y}}(t)+\frac{\tau^{2}}{2} \ddot{\bar{y}}(t)+\tau^{3} \Delta_{1}(t, \tau) \\
& \bar{y}_{v}(t-\tau)=\bar{y}_{v}(t)-\tau \dot{\bar{y}}_{v}(t)+\tau^{2} \Delta_{2}(t, \tau)
\end{aligned}
$$

The accuracy of the estimate of $y_{v}$ is given as follows.

$$
\begin{aligned}
\widehat{\bar{y}}(t)= & \frac{1}{n \varepsilon} \int_{0}^{n \varepsilon}\left(\bar{y}(t)-\tau \dot{\bar{y}}(t)+\frac{1}{2} \tau^{2} \ddot{\bar{y}}(t)+\tau^{3} \Delta_{1}(t, \tau)\right) \mathrm{d} \tau \\
& +\frac{1}{n} \int_{0}^{n \varepsilon}\left(\bar{y}_{v}(t)-\dot{\bar{y}}_{v}(t) \tau+\tau^{2} \Delta_{2}(t, \tau)\right) S\left(\frac{t-\tau}{\varepsilon}\right) \mathrm{d} \tau+\varepsilon^{2} \mathbb{V}\left(\frac{t}{\varepsilon}\right) \\
= & \bar{y}\left(t-\frac{n \varepsilon}{2}\right)+\varepsilon^{2}\left(\frac{n^{2}}{24} \ddot{\bar{y}}(t)+\dot{\bar{y}}_{v}(t) \mathbb{S}\left(\frac{t}{\varepsilon}\right)\right)+\varepsilon^{2} \mathbb{V}\left(\frac{t}{\varepsilon}\right)+\mathcal{O}\left(\varepsilon^{3}\right), \\
\left(\frac{1}{n \varepsilon} \int_{t-n \varepsilon}^{t} S^{2}\left(\frac{\mu}{\varepsilon}\right) \mathrm{d} \mu\right) \cdot \widehat{y}_{v}(t)= & \frac{1}{\varepsilon} \frac{1}{n \varepsilon} \int_{t-n \varepsilon}^{t}\left[\bar{y}(\mu)+\varepsilon y_{v}(\mu) S\left(\frac{\mu}{\varepsilon}\right)+\varepsilon^{2} \nu-\bar{y}\left(\mu-\frac{n \varepsilon}{2}\right)\right. \\
& \left.-\varepsilon^{2}\left(\frac{n^{2}}{24} \ddot{\bar{y}}(\mu)+\dot{\bar{y}}_{v}(\mu) \mathbb{S}\left(\frac{\mu}{\varepsilon}\right)\right)-\varepsilon^{2} \mathbb{V}\left(\frac{\mu}{\varepsilon}\right)+\mathcal{O}\left(\varepsilon^{3}\right)\right] S\left(\frac{\mu}{\varepsilon}\right) \mathrm{d} \mu \\
= & \left(\frac{1}{n \varepsilon} \int_{t-n \varepsilon}^{t} S^{2}\left(\frac{\mu}{\varepsilon}\right) \mathrm{d} \mu\right) \cdot y_{v}(t)+\varepsilon \mathfrak{V}(t)+\mathcal{O}\left(\varepsilon^{2}\right) \cdot \mathbb{1}_{p},
\end{aligned}
$$

where $\varepsilon \mathfrak{V}=\int_{t-n \varepsilon}^{t}(\nu(\mu / \varepsilon)-\mathbb{V}(\mu / \varepsilon)) \cdot S(\mu / \varepsilon) \mathrm{d} \mu$, in which the norm of $\mathfrak{V}$ is independent of $\varepsilon$. $\mathbb{S}(\cdot)$ is the primitive of $S$ with zero mean, and $\mathbb{V}$ is the primitive of $\nu$, that is

$$
\mathbb{S}\left(\frac{t}{\varepsilon}\right)=\frac{1}{n} \int_{\frac{t}{\varepsilon}-n}^{\frac{t}{\varepsilon}} \mu S(\mu) \mathrm{d} \mu .
$$

The last equation is obtained by

$$
\begin{aligned}
& \frac{1}{n \varepsilon^{2}} \int_{t-n \varepsilon}^{t}-\varepsilon^{2} \dot{\bar{y}}_{v}(\mu) \mathbb{S}\left(\frac{\mu}{\varepsilon}\right) S\left(\frac{\mu}{\varepsilon}\right) \mathrm{d} \mu \\
= & -\frac{\varepsilon^{2}}{2} \dot{\bar{y}}_{v}\left(\mathbb{S}^{2}\left(\frac{t}{\varepsilon}\right)-\mathbb{S}^{2}\left(\frac{t-n \varepsilon}{\varepsilon}\right)\right)+\mathcal{O}\left(\varepsilon^{2}\right) \cdot \mathbb{1}_{p} \\
= & \mathcal{O}\left(\varepsilon^{2}\right) \cdot \mathbb{1}_{p} .
\end{aligned}
$$

Define $\mathfrak{p}=1 /(n \varepsilon) \int_{t-n \varepsilon}^{t} S^{2}(\mu / \varepsilon) \mathrm{d} \mu>0$, which is independent of $\varepsilon$ and $t$ due to the periodic property of $S$. Therefore, the accuracy of the estimator is

$$
\widehat{y}_{v}(t)=y_{v}+\varepsilon \mathfrak{p}^{-1} \cdot \mathfrak{V}(t)+\mathcal{O}\left(\varepsilon^{2}\right) \cdot \mathbb{1}_{p} .
$$

For a given $n, \mathfrak{V}$ is bounded in the form of $\omega(x, t)$. In this design, the periodic term $\varepsilon \kappa\left(x, \frac{t}{\varepsilon}\right)$ equals to zero. 


\section{Appendix C. Proof of Proposition 4}

For the asymptotic case, according to the converse Lyapunov theorem (Theorem 4.16 in [16]), there is a smooth, positive definite function $V(|\underline{\chi}|)$ that satisfies the following inequalities,

$$
\begin{aligned}
& \alpha_{1}(|\underline{\chi}|) \leq V(t, \underline{\chi}) \leq \alpha_{2}(|\underline{\chi}|) \\
& \alpha_{3}(|\underline{\chi}|) \geq \nabla_{t} V+\nabla_{\underline{\chi}} V^{\top} \mathbf{F}\left(\underline{x}, \underline{x}_{c}\right),
\end{aligned}
$$

where $\alpha_{i}(i=1, \ldots, 3)$ are class $\mathcal{K}$ functions. For $\underline{\chi} \in \mathcal{D}$ with any compact set $\mathcal{D}$, there exists a class $\mathcal{K}$ function such that

$$
\left|\nabla_{\underline{\chi}} V(t, \underline{\chi})\right| \leq \alpha_{4}(|\underline{\chi}|)
$$

For $\bar{\chi} \in \mathcal{D}$ and a given $\varepsilon$, there always exists $\varepsilon^{(3)}$ such that $\varepsilon|\Delta| \leq \varepsilon^{(3)}$. Define a mapping $r: \varepsilon \mapsto \mathbb{R}_{+}$ satisfying $\varepsilon^{(3)}<\alpha_{3}\left(\alpha_{2}^{-1}\left(\alpha_{1}(r)\right)\right) \cdot \alpha_{4}^{-1}(r)$. In terms of Lemma 9.3 in [16] the solution $\bar{\chi}$ of the averaged system satisfies that, for some class $\mathcal{K} \mathcal{L}$ function $\mathfrak{B}$, there exist a time $T$ such that

$$
|\bar{\chi}(t)| \leq \mathfrak{B}\left(|\bar{\chi}(0)|, t-t_{0}\right), \quad \forall t \in[0, T)
$$

and

$$
|\bar{\chi}(t)| \leq \alpha_{1}^{-1}\left(\alpha_{2}\left(\alpha_{3}^{-1}\left(\varepsilon^{(3)} \alpha_{4}(r(\varepsilon))\right)\right)\right):=\mathfrak{D}(\varepsilon), \quad \forall t \geq T
$$

where the function $\mathfrak{D}(\cdot)$ is class $\mathcal{K}$.

The behaviour analysis of the actual system follows the one of exponential case, completing the proof. 